Bull. Mater. Sci., Vol. 22, No. 5, August 1999, pp. 891-900. (C) Indian Academy of Sciences.

\title{
Numerical simulations of fracture initiation in ductile solids under mode I, dynamic loading
}

\author{
R NARASIMHAN* and S BASU ${ }^{\dagger}$ \\ Department of Mechanical Engineering, Indian Institute of Science, Bangalore 560 012, India
}

\begin{abstract}
In this paper, an overview of some recent computational studies by the authors on ductile crack initiation under mode $I$, dynamic loading is presented. In these studies, a large deformation finite element procedure is employed along with the viscoplastic version of the Gurson constitutive model that accounts for the micro-mechanical processes of void nucleation, growth and coalescence. A three-point bend fracture specimen subjected to impact, and a single edge notched specimen loaded by a tensile stress pulse are analysed. Several loading rates are simulated by varying the impact speed or the rise time and magnitude of the stress pulse. A simple model involving a semi-circular notch with a pre-nucleated circular hole situated ahead of it is considered. The growth of the hole and its interaction with the notch tip, which leads to plastic strain and porosity localization in the ligament connecting them, is simulated. The role of strain-rate dependence on ductile crack initiation at high loading rates, and the specimen geometry effect on the variation of dynamic fracture toughness with loading rate are investigated.
\end{abstract}

Keywords. Ductlle fracture; dynamic loading; strain-rate dependence; finite elements.

\section{Introduction}

Ductile fracture occurs on the micro-scale by nucleation, growth and coalescence of voids. The nucleation of voids occurs mainly by brittle cracking or interfacial decohesion of inclusions. This is followed by growth of the voids which is caused by plastic deformation in the surrounding material, and is strongly influenced by high triaxial tension. It has been observed by Cox and Low (1974) that in structural materials, such as steels and aluminium alloys, void initiation can involve two distinct population of inclusions. In such materials, voids initiate first at larger inclusions, and after growing to some size, the voids coalesce, or link up with a nearby crack tip via a void sheet comprised of voids nucleated from smaller particles.

The fracture mechanics concepts, employed for designing structural components, are generally based on the static fracture toughness. However, in many engineering applications, highly dynamic loading may be encountered. These include failure of metallic armour by projectile impact, explosive detonation, pressurized thermal shocks in nuclear reactors, etc. For many metallic materials, the dynamic fracture toughness depends strongly on the loading rate measured in terms of $\dot{K}$ or $j$ (Priest 1976; Owen et al 1997). Several factors, such as material inertia, strain-rate sensitivity, thermal softening caused by adiabatic heating, matrix-inclusion bond strength, etc may influence the ductile fracture processes,

*Author for correspondence and, hence, the functional relationship between the dynamic fracture toughness and $\boldsymbol{j}$ is required. A quantitative understanding of these effects will help in developing materials which are better suited to resist dynamic fracture.

Furthermore, it is important to investigate whether a fracture criterion of the form

$$
J_{\text {applied }}(t)=J_{\mathrm{c}}(j),
$$

has a universal character (i.e. independent of specimen geometry). This would justify application of the above criterion for dynamic ductile crack initiation. In the above expression, $J_{\text {applied }}$ refers to the value of $J$ integral obtained from applied boundary conditions, $J_{c}$ is the dynamic fracture toughness which is postulated to be a function of average loading rate $j=J_{\mathrm{c}} t_{\mathrm{f}}$, where $t_{\mathrm{f}}$ is the time to fracture. In this paper, an overview of salient results obtained from a series of recent finite element investigations by the authors (Basu and Narasimhan 1996, $1999 \mathrm{a}, \mathrm{b})$, where the above issues have been addressed, is presented.

In the simulations reported here, the viscoplastic version of the Gurson constitutive model (Pan et al 1983), which accounts for the ductile failure processes of microvoid nucleation, growth and coalescence, is used within the framework of a finite deformation theory with small elastic strains. This model was employed earlier by Tvergaard and Needleman (1988), and Needleman and Tvergaard (1991) on dynamic, ductile fracture. A single edge notched specimens (SEN(T)), loaded by a tensile stress pulse, and a three-point bend specimen (TPB) 
subjected to impact are analysed under 2D, plane strain conditions. Several loading rates are simulated by varying the impact speed or the rise time and magnitude of the stress pulse. A simple model involving a semi-circular notch with a pre-nucleated large circular void situated ahead of it is considered. In addition, a plastic-straincontrolled void nucleation law is employed within the framework of the Gurson model to represent micro-void nucleation at small-sized particles (less than, say, $1 \mu \mathrm{m}$ in size) which have been assumed to be uniformly distributed within the matrix. Fracture initiation is assumed to occur when the entire ligament connecting the notch and the large void experiences failure owing to micro-void coalescence.

The accumulation of porosity in the ligament connecting the notch with the void, and the growth of the void, are carefully examined in order to address the issues outlined earlier. A direct comparison is made between the results for rate-independent and rate-dependent material behaviour, and the role of rate dependence on the ductile fracture processes at high loading rates is assessed. Further, the evolution histories of void growth near the notch tip (with respect to $J$ ) in the TPB and SEN(T) specimens at the same average loading rate $j$ are compared. This sets the stage for discussing the locus of fracture toughness, $J_{c}$, with $\dot{j}$ obtained from the micromechanical modelling of the two specimens. Several factors, such as the universal character of the above locus, effect of loading rate, and rate dependence on $J_{c}$, are examined.

\section{Constitutive model}

In this work, the viscoplastic version of the Gurson constitutive model, which was introduced by Pan et al (1983), is employed. This model characterizes porosity in the material in terms of an internal variable $f$, the void volume fraction. The viscoplastic potential function depends on the macroscopic (aggregate) Cauchy stress tensor $\sigma_{i j}$, the matrix (microscopic) tensile flow stress $\sigma_{\mathrm{m}}$, and the void volume $f$ and is given by:

$$
\begin{gathered}
\Phi\left(\sigma_{i j}, \sigma_{\mathrm{m}}, f\right)=\frac{\sigma_{\mathrm{c}}^{2}}{\sigma_{\mathrm{m}}^{2}}+2 f^{*} q_{1} \cosh \left(\frac{3 \sigma_{\mathrm{H}}}{2 \sigma_{\mathrm{m}}}\right) \\
-\left(1+\left(q_{1} f^{*}\right)^{2}\right)=0 .
\end{gathered}
$$

Here, $\sigma_{m}$ is the tensile flow strength of the matrix material. The macroscopic equivalent stress $\sigma_{e}$, and the hydrostatic stress $\sigma_{\mathrm{H}}$ are defined by,

$$
\sigma_{e}=\sqrt{\frac{3}{2} s_{i j} s_{i j}},
$$

and

$$
\sigma_{\mathrm{H}}=\frac{1}{3} \sigma_{k k},
$$

where $S_{i j}=\sigma_{i j}-\sigma_{\mathrm{H}} \delta_{i j}$ is the stress deviator.

The parameter $q_{1}$ in (2) was introduced by Tvergaard (1981, 1982), with a suggested value of 1.5 , to obtain better agreement between the predictions of the above model and numerical studies on periodic array of voids. The function $f^{*}(f)$ was proposed by Tvergaard and Needleman (1984) to account for rapid evolution of $f$ due to void coalescence near failure and is given by:

$$
f^{*}=\left\{\begin{array}{ll}
f & f \leq f_{\mathrm{c}} \\
f_{\mathrm{c}}+K\left(f-f_{\mathrm{c}}\right) & f>f_{\mathrm{c}}
\end{array}\right\},
$$

where $K=\left(1 / q_{1}-f_{\mathrm{c}}\right) /\left(f_{\mathrm{F}}-f_{\mathrm{c}}\right)$. Here, $f_{\mathrm{c}}$ is the value of the void volume fraction at which void coalescence commences and $f_{\mathrm{F}}$ its value at final failure. From (2), it is evident that as $f \rightarrow f_{\mathrm{F}}, f^{*} \rightarrow 1 / q_{1}$ and the material looses all its stress-carrying capacity. Thus, an essential feature of this model is that a failure criterion is directly built in to the constitutive equations. An estimate of $f_{\mathrm{c}}$ obtained by Brown and Embury (1973), based on a simple model, was 0.15 . Also, a numerical investigation by Andersson (1977) suggested that $f_{\mathrm{F}} \simeq 0 \cdot 25$. The above values of $f_{\mathrm{c}}$ and $f_{\mathrm{F}}$ have been employed in the simulations reported here.

In general, the evolution of the void volume fraction is due to growth of the existing voids as well as due to nucleation of the new voids and, hence,

$$
\dot{f}=\dot{f}_{\text {growth }}+\dot{f}_{\text {nucleation }} \text {. }
$$

The growth law is described by,

$$
\dot{f}_{\text {growth }}=(1-f) D_{k k}^{p}
$$

where $D_{i j}^{p}$ is the plastic part of the rate of deformation tensor. This relation is an outcome of the plastic incompressibility of the matrix material. In this work, a plastic-strain-controlled void nucleation law has been used to model micro-void nucleation at small-sized particles (less than, say, $1 \mu \mathrm{m}$ in size), as suggested by Thomason (1990), which are assumed to be uniformly distributed in the matrix. Thus,

$$
\dot{f}_{\text {nuckeation }}=\Re \dot{\varepsilon}_{\mathrm{m}}^{p}
$$

where, $\mathscr{T}($.$) is a function of the matrix plastic strain \varepsilon_{\mathrm{m}}^{p}$. The function $\mathscr{T}\left(\varepsilon_{\mathrm{m}}^{p}\right)$ was chosen as (Chu and Needleman 1980):

$$
\pi=\frac{f_{\mathrm{n}}}{s_{\mathrm{n}} \sqrt{2 \pi}} \exp \left[-\frac{1}{2}\left(\frac{\varepsilon_{\mathrm{m}}^{p}-\varepsilon_{\mathrm{n}}}{s_{\mathrm{n}}}\right)^{2}\right]
$$


so that void nucleation follows a normal distribution about a mean nucleation strain $\varepsilon_{n}$, and with a standard deviation $s_{\mathrm{n}}$. In the above equation, $f_{\mathrm{n}}$ denotes the volume fraction of the uniformly distributed small-sized particles. In the analyses reported here, $f_{\mathrm{n}}, \varepsilon_{\mathrm{n}}$ and $s_{\mathrm{n}}$ are assumed as $0.04,0.3$ and 0.1 , respectively. In addition, a prenucleated large void (comparable in size to the initial notch diameter) ahead of the notch tip has been considered (see \& 3 ). However, the actual process of initiation of this large void by interfacial decohesion around a second-phase particle (Argon et al 1975) is not modelled in this work.

The matrix material is characterized as a viscoplastic solid (i.e. one that displays flow stress elevation at high strain rates), and the matrix plastic strain-rate $\dot{\varepsilon}_{\mathrm{m}}^{p}$ is given by:

$$
\dot{\varepsilon}_{\mathrm{m}}^{p}=\dot{\varepsilon}_{0}\left(\frac{\sigma_{\mathrm{m}}}{g\left(\varepsilon_{\mathrm{m}}^{p}\right)}\right)^{1 / \mathrm{m}} .
$$

Here, $\dot{\varepsilon}_{0}$ is a reference strain-rate and ' $m$ ' a rate exponent. The function $g\left(\varepsilon_{\mathrm{m}}^{p}\right)$ represents the effective tensile flow stress of the matrix material in a tensile test carried out at a strain rate such that $\dot{\varepsilon}_{\mathrm{m}}^{p}=\dot{\varepsilon}_{0}$. In this work, an isotropic power law hardening material with a strain hardening exponent $N$ is considered, so that,

$$
g\left(\varepsilon_{\mathrm{m}}^{p}\right)=\sigma_{0}\left(\frac{\varepsilon_{\mathrm{m}}^{p}}{\varepsilon_{0}}+1\right)^{N}
$$

Here, $\sigma_{0}$ is the initial yield stress and $\varepsilon_{0}=\sigma_{0} / E, E$ being the Young's modulus, is the yield strain. It should be noted from (8) that in the limit as the strain-rate exponent $\mathrm{m} \rightarrow 0$, the present viscoplastic material model reduces to the rate-independent Gurson model. Thus, the modelling of slow, quasi-static loading (when the viscoplastic material will not display any flow stress elevation) as well as rate-independent behaviour under dynamic loading can be accomplished by using a very small value for $\mathrm{m}$. In the simulations to be reported in $\S 4$, the material properties assumed for the rate-independent material are: $m=0.005$, $\sigma_{0}=400 \mathrm{MPa}, E=200 \mathrm{GPa}, \quad \nu=0.3, \quad N=0.1$ and $\rho_{0}$ $($ density $)=7800 \mathrm{~kg} / \mathrm{m}^{3}$. These properties correspond to a typical intermediate strength steel. For simulating ratedependent material behaviour, $\mathrm{m}$ and $\dot{\varepsilon}_{0}$ are considered as 0.05 and $0.002 \mathrm{~s}^{-1}$, respectively.

Equations (2) to (8) are used within the framework of a finite deformation elastic-viscoplastic theory with small elastic strains (Needleman and Tvergaard 1987). The final constitutive equations are derived by following the rate tangent modulus method proposed by Pierce et al (1984).

As noted earlier, complete loss of material stresscarrying capacity occurs when $f^{*}=1 / q_{1}$ (or, equivalently, when $f=f_{\mathrm{F}}$ ), resulting in local material failure. This

implies that the material should completely separate at this point and a traction-free surface develop. This failure criterion was implemented in the numerical simulation by freezing the evolution of $f$ after it acquired a value close to $f_{\mathrm{F}}$ (around $0.95 f_{\mathrm{F}}$ ). The macroscopic material response is then elastic-perfectly plastic with a small pressuredependent yield stress. The condition $f=0.95 f_{\mathrm{F}}$ was used instead of $f=f_{\mathrm{F}}$, since as $f \rightarrow f_{\mathrm{F}}$, the macroscopic equivalent stress $\sigma_{e} \rightarrow 0$, resulting in numerical difficulties.

\section{Computational aspects}

The single edge notched specimen subjected to tensile loading (SEN(T)), and the three point bend specimen (TPB) that are analysed in this work are shown in figures $1 \mathrm{a}$ and $\mathrm{b}$, respectively. The dimensions of both the specimens are: length $2 L=160 \mathrm{~mm}$, width $W=40 \mathrm{~mm}$ and crack length $c=20 \mathrm{~mm}$. The SEN(T) specimen was loaded by applying a uniform tensile stress pulse $\sigma_{\infty}(t)$ to the top edge of the specimen. This function is chosen in the form:

$$
\sigma_{\infty}(t)=\left\{\begin{array}{ll}
\hat{\sigma} t / t_{\mathrm{r}} & \text { for } t \leq t_{\mathrm{r}} \\
\hat{\sigma} & \text { for } t>t_{\mathrm{r}}
\end{array} .\right.
$$

Here, $t_{\mathrm{r}}$ represents a rise time, and $\hat{\sigma}$ the peak stress attained by the tensile loading. By varying $t_{\mathrm{r}}$ and $\hat{\sigma}, a$ range of loading rates, $j$ (from $10^{4}$ to $10^{7} \mathrm{kN} / \mathrm{m}$-s), was generated at the notch tip. The TPB specimen is subjected to impact (at a speed $V_{0}$ ) (figure $1 \mathrm{~b}$ ). By varying $V_{0}$, different loading rates are generated in this specimen. In the finite element analyses, only half the specimen (on one side of the crack plane) is modelled because of presence of symmetry in the case of TPB, whereas for SEN(T), the complete specimen is modelled, since wave propagation effects render this specimen unsymmetric.

(a)
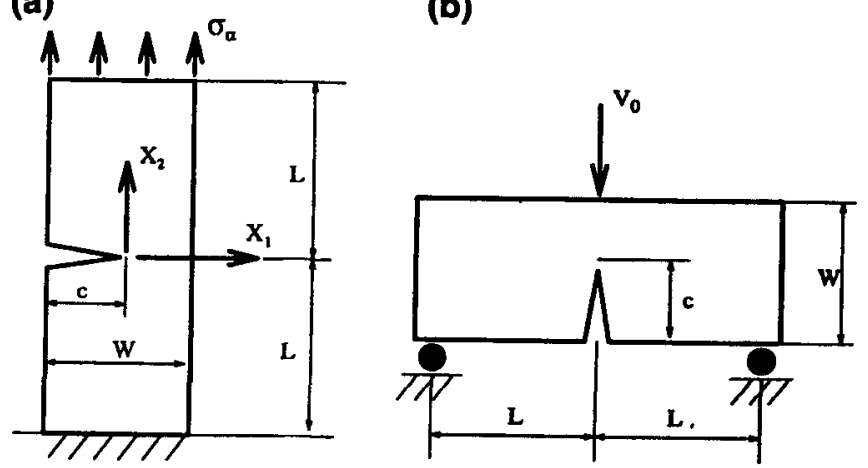

Figure 1. Schematic diagram of a. the SEN(T), and b. TPB specimens. 
The finite element mesh used to represent the SEN(T) specimen is shown in figure 2a. This mesh comprises of 1112 four-noded (2-D plane strain) quadrilateral elements, with 2396 degrees of freedom. In figure $2 b$, the details of the refined mesh which is employed near the notch tip is displayed. The initial notch diameter, $b_{0}$ is $0.05 \mathrm{~mm}$. At a distance $L_{0}=0.25 \mathrm{~mm}$, ahead of the notch

(a)

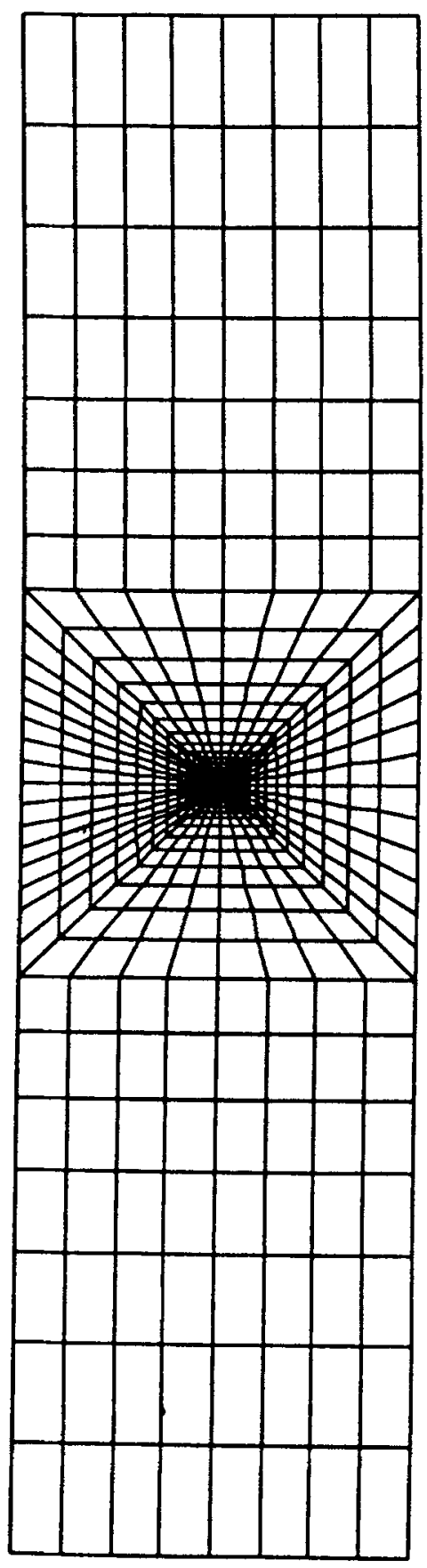

tip, a circular void of diameter $a_{0}=0.05 \mathrm{~mm}$ is placed (figure $2 \mathrm{~b}$ ). The objective in employing this model is to simulate the interaction between a void (which had nucleated, say, around a large second-phase particle or inclusion) and the notch tip. Fracture initiation is assumed to occur here when the entire ligament connecting the notch and hole experiences failure by micro-void coalescence. Hence, in this work, the spacing $L_{0}$ between the notch and hole is considered as an important length scale of physical significance. The hole is placed directly ahead of the tip in figure 2b, since Aravas and McMeeking (1985) had noted that the growth rate of a hole located directly ahead of the notch tip is higher than that situated at an angle with respect to the notch line. The average dimension of the smallest element (near the notch tip and the inclusion) in figure $2 \mathrm{~b}$ is approximately $0.016 \mathrm{~mm}$. A similar model is employed in the study of the TPB specimen as well.

The finite element procedure used in this work is based on an updated Lagrangian formulation that accounts for finite deformations and rotations (Narasimhan 1994). The central difference scheme is used to integrate the equations of motion in the dynamic analyses. A time-step size of $0.5 \times 10^{-9} \mathrm{~s}$ is employed in these analyses, which is sufficient to ensure that the numerical solution remains stable. Also, the stability of the numerical solution in the dynamic analyses is continuously monitored by checking the global energy balance, as suggested by Belytshko (1981) for nonlinear problems. A domain integral version of $J$ proposed by Nakamura $e t$ al (1986), which is appropriately modified for finite deformation, is employed to compute $J$ from the finite element results.

(b)

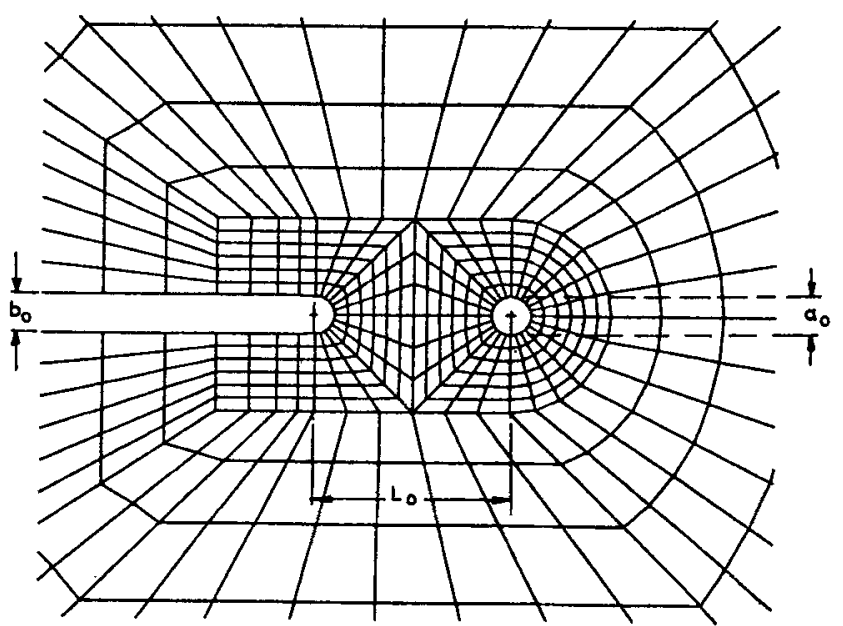

Figure 2. a. Typical mesh used in finite element analysis of the SEN(T) specimen. b. Details of near-tip mesh showing the notch and the pre-nucleated hole. 


\section{Results and discussion}

\subsection{Plastic strain localization and porosity distribution}

The evolution of porosity is intimately related to the development of high hydrostatic tension and to plastic strain localization. It is well known (Hutchinson 1968) that hydrostatic tension ahead of the notch tip under Mode I plane strain is very high. In the present simulations, coalescence of the void with the notch was considered to occur via a void sheet (Cox and Low 1974) rather than due to direct impingement. The earlier theories, which had not considered evolution of porosity, tried to formulate approximate relations, connecting the void, ligament and notch dimensions, that had to be satisfied at a point when sudden coalescence became imminent. For example, such relations had been proposed in the studies by Rice and Johnson (1970), and Brown and Embury (1973). However, the present approach obviates the need for using such relations, since phenomena like intense strain localization and consequent weakening of the ligament with development of porosity have been accounted for in a natural way by the constitutive equations explained in $\S 2$.

The contours of matrix plastic strain $\varepsilon_{m}^{p}$ at two time instants, obtained from the rate-independent dynamic analysis of the TPB specimen with impact speed $V_{0}=10 \mathrm{~m} / \mathrm{s}$, are presented in figures $3 a$ and $b$. At the stage represented in figure 3a, the notch blunts to a diameter of $b=2 \cdot 9 b_{0}$ and the longitudinal hole diameter attains a value of $a=1.75 a_{0}$. At the stage pertaining to figure $3 b$, the notch diameter blunts to a diameter of $b=3.4 b_{0}$, and the hole diameter attains a value of $a=2 \cdot 2 a_{0}$.

The above figures show the localization of plastic strain in the ligament. Figure $3 \mathrm{a}$ shows that while the regions of high plastic strain have developed around the hole as well as the notch, the plastic strain in the middle of the ligament is still small. Figure $3 b$ shows that these regions of high plastic strain move towards each other, thereby bridging the ligament. Thus, it is apparent that the approach adopted in this work is more realistic in contrast to placing a void in the strain field of a blunting crack, since, as can be seen from figures $3 a$ and $b$, the void strongly contributes towards modifying the local strain field ahead of the notch. In fact, growth of the void and its interaction with the notch ultimately leads to plastic strain localization in the ligament (figure $3 \mathrm{~b}$ ).

The narrow band of plastic strain localization in the ligament in figure $3 \mathrm{~b}$, results in the development of porosity inside it, while the regions outside remain relatively free from porosity. To understand this, the contours of void volume fraction, $f$, at the same time instants as shown in figures $3 a$ and $b$ are displayed in figures $4 a$ and $b$. Figure $4 a$ reveals that while there is a high level of porosity formation near the notch tip and hole, the value of $f$ in the middle of the ligament is much smaller. In other words, while the contours of void volume fraction near the hole spread back towards the notch, those near the notch spread forward towards the hole.

The accumulation of porosity near the notch and the hole (figure 4a) is due to large plastic strain concentration (compare figure $4 a$ with $3 a$ ), and high triaxiality. The regions within contour $D$ (shown hatched in figure 4a) have experienced material failure (i.e. $f \simeq 0.95 f_{F}$ ). In other words, these are the regions that have lost the stress-carrying capacity, and can be treated as an extension of either the void or the crack, depending upon their points of emanation. Figure $4 \mathrm{~b}$ further reveals that towards the later stage of deformation, the contours of high void volume fraction emanating from the hole and the notch have linked up with each other. The region within contour $D$ (shown hatched in figure $4 \mathrm{~b}$ ), which encompasses the entire ligament between the notch and

(a)

CONTOUR LEVELS

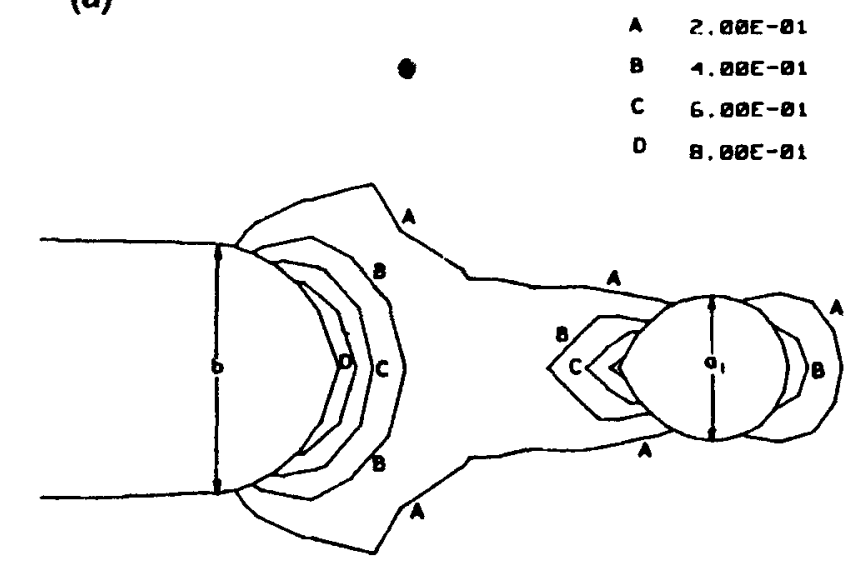

(b)

CONTOUR LEVELS

$$
\begin{array}{ll}
\text { A } & 2.00 E-01 \\
\text { B } & 4.00 E-01 \\
\text { C } & 6.00 E-01 \\
\text { D } & 8.00 E-01
\end{array}
$$

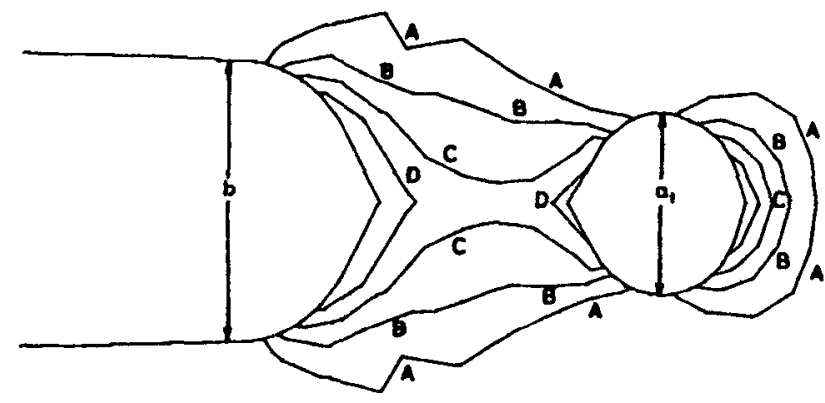

Figure 3. Contours of matrix plastic strain obtained from the dynamic analysis of the TPB specimen corresponding to impact speed of $10 \mathrm{~m} / \mathrm{s}$ at the stage when a. the notch has blunted to a diameter of $b=2.9 b_{0}$, and $b$. when $b=3.4 b_{0}$. 
the hole, has experienced material failure by micro-void damage.

There is a strong similarity between the contours of matrix plastic strain shown in figures $3 a$ and $b$ and those of void volume fraction displayed in figures $4 a$ and $b$. At the stages represented in figures $3 a$ and $4 a$, the central portion of the ligament (within contour $A$ in figure $3 a$ ) begins to undergo plastic-strain-controlled void nucleation. This leads to rapid failure of the ligament, as can be seen in figure $4 \mathrm{~b}$. The above phenomenon thus corresponds to the classic void sheet mechanism, which was observed in experimental studies by Cox and Low (1974) involving materials with two sets of voidnucleating particles of vastly different sizes.

The hatched region in figure $4 b$, within which failure by void sheeting has occurred is a narrow band that bridges the entire ligament between the notch tip and the hole. It should however be noted here that concentrations of void volume fraction contours, encircling discrete points (representing the small scale particles) within this band, are not perceived in figure $4 \mathrm{~b}$. This is because, as mentioned in $\$ 2$, the small-scale particles (conceivably, less than $1 \mu \mathrm{m}$ in size) are assumed to be uniformly dispersed in the matrix. An explicit modelling of a statistically significant number of these tiny particles, in a manner such that their discrete character is preserved, would perhaps require an extremely fine discretization, with its consequent implication on the computational requirements. However, the important physical processes of strain concentration in the ligament, after the notch and hole have deformed to some extent and its consequent failure by porosity formation, which are involved in void sheeting, have been well represented in the present simulations. This is a direct outcome of the use of the Gurson constitutive equations (in particular, the micro-void nucleation law, (6) and (7)).

\subsection{Effect of strain-rate dependence and loading rate on ductile crack initiation}

In figures $5 \mathrm{a}$ and $\mathrm{b}$, the variations of void volume fraction $f$ versus normalized distance $X / L_{0}$ along the ligament have been plotted corresponding to different values of $J /\left(\sigma_{0} L_{0}\right)$, for both the rate-independent and rate-dependent analyses, respectively. These analyses pertain to the TPB specimen with impact speed $V_{0}=8 \mathrm{~m} / \mathrm{s}$. In this plot as well as in other similar plots, presented later, the distance $X$ is measured from the centre of curvature of the notch in the undeformed configuration. Comparison of figures $5 \mathrm{a}$ and $b$, show that porosity accumulates much more rapidly for the rate-independent case. Thus, it can be noted from figure 5a that at $J /\left(\sigma_{0} L_{0}\right)=0.71$, a substantial portion of the ligament in the rate-independent simulation has lost its stress-carrying capacity. In contrast, for the ratedependent simulation, the porosity throughout the ligament is less than the pre-set coalescence limit, $f_{c}=0.15$, even at $J /\left(\sigma_{0} L_{0}\right)=0.91$.

The evolution of the normalized transverse hole opening $\left(a-a_{0}\right) / a_{0}$ with respect to normalized $J$ for different impact speeds is plotted in figures $6 a$ and $b$ for the rate-independent and rate-dependent analyses (of the TPB specimen), respectively. It can be seen that at a fixed $J /\left(\sigma_{0} L_{0}\right)$, there is a decrease in the normalized hole opening, as the impact speed increases from 2 to $8 \mathrm{~m} / \mathrm{s}$. This effect is more predominant for the rate-independent simulation (figure 6a) compared to the rate-dependent case (figure $6 \mathrm{~b}$ ). However, with further increase in the impact speed (from 8 to $20 \mathrm{~m} / \mathrm{s}$ ), the reduction in hole opening at a given $J /\left(\sigma_{0} L_{0}\right)$, is comparatively less for the rate-independent simulation. It should be noted that in the rate-independent simulations, inertial effects are solely responsible for slowing down the rate of growth of the hole, with respect to $J$. On the other hand, in the ratedependent simulations, inertial and strain rate sensitivity

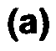

CONTOUR LEVELS
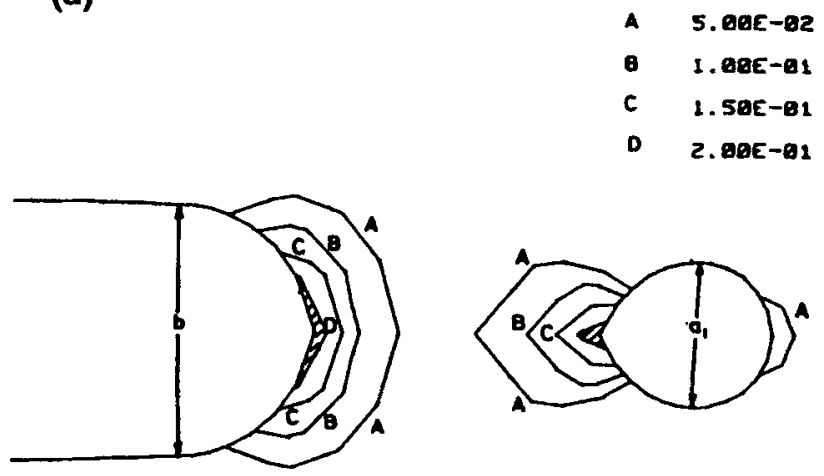

(b)

CONTOUR LEVELS

$$
\begin{array}{ll}
\text { A } & 5.00 \varepsilon-02 \\
\text { B } & 1.00 \varepsilon-01 \\
\text { C } & 1.50 E-01 \\
\text { D } & 2.00 E-01
\end{array}
$$

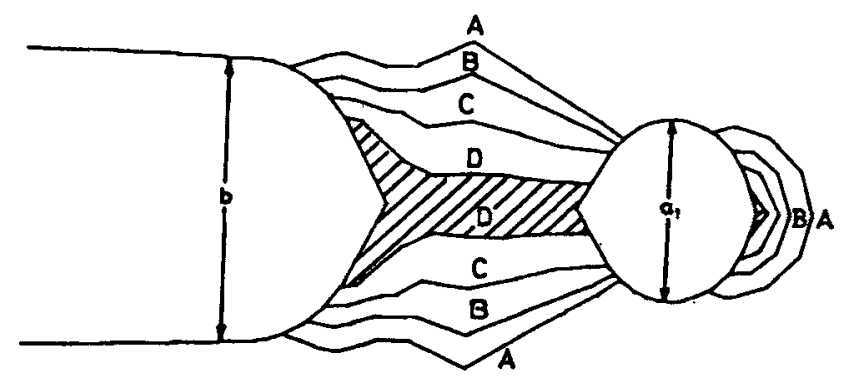

Figure 4. Contours of void volume fraction obtained from the dynamic analysis of the TPB specimen corresponding to impact speed of $10 \mathrm{~m} / \mathrm{s}$ at the stage when a. the notch has blunted to a diameter of $b=2 \cdot 9 b_{0}$, and $\mathrm{b}$. when $b=3 \cdot 4 b_{0}$. 
effects combine to further decrease the growth rate of the hole as the impact speed increases from 8 to $20 \mathrm{~m} / \mathrm{s}$, as can be seen from figure $6 \mathrm{~b}$.

On comparing the curves pertaining to the same impact speed in figure $6 \mathrm{a}$ with figure $6 \mathrm{~b}$, it can be seen that strain rate sensitivity causes considerable slowing down of the growth rate of the hole with respect to the normalized loading parameter, $J /\left(\sigma_{0} L_{0}\right)$. This observation is corroborated by the results of Budiansky et al (1982) on the growth of a spherical void in a nonlinearly viscous solid, which follows an uniaxial relation similar to what has been described in (8). It can be observed from figures $5.1(a, b)$ of their paper that the dilation rate of a spherical void decreases as the rate exponent $m$ increases, and that this effect is more pronounced at higher hydrostatic stress levels that prevail ahead of the blunting notch.

In figures $7 \mathrm{a}$ and $\mathrm{b}$, the variation of $f$ in the ligament for static and rate-independent dynamic analyses of the
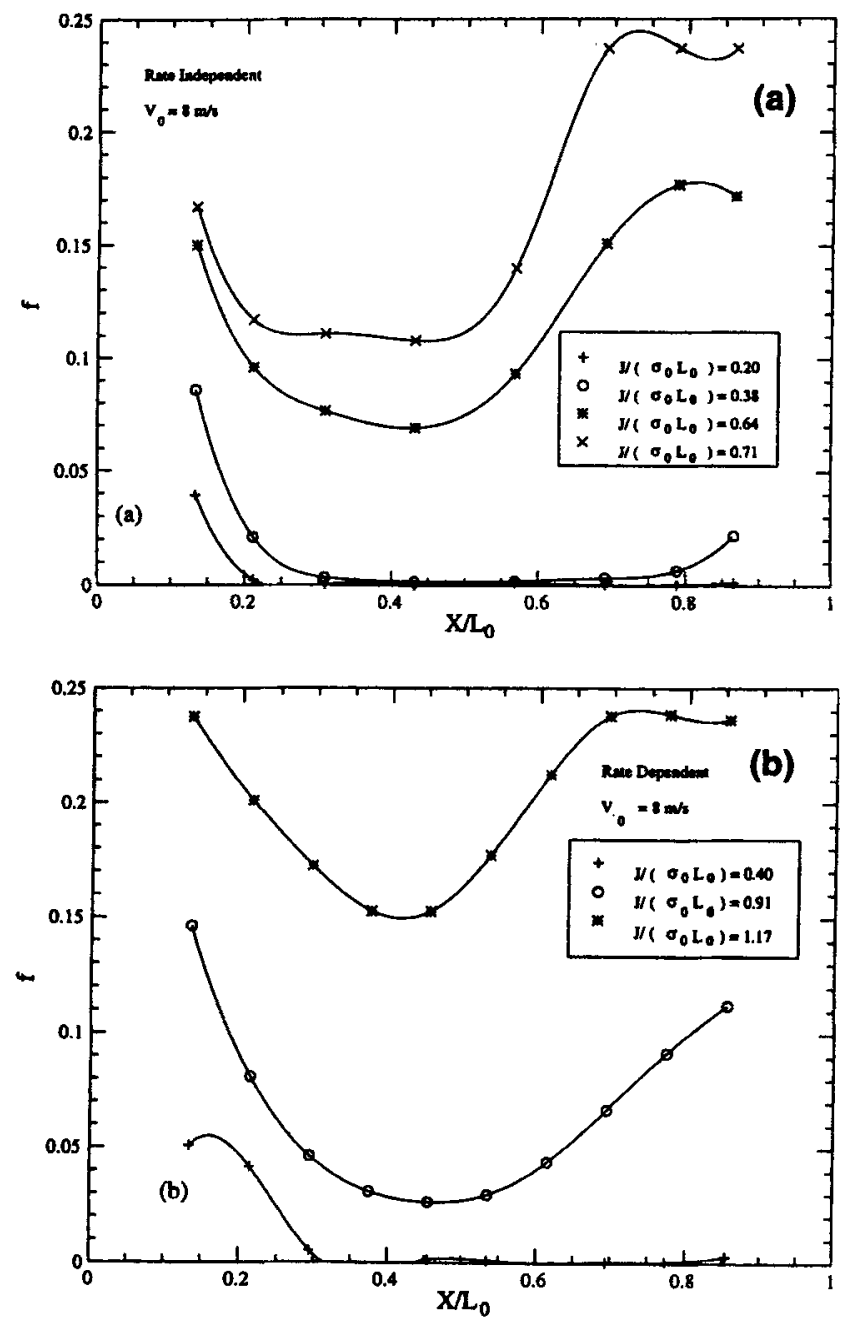

Figure 5. Distribution of void volume fraction, $f$, in the ligament connecting the notch tip and the hole, obtained from dynamic analysis of the TPB specimen (at $V_{0}=8 \mathrm{~m} / \mathrm{s}$ ), pertaining to a. rate-independent case, and b. rate-dependent case.
SEN(T) specimen are shown. The plots in these figures correspond to different values of the normalized loading parameter $J /\left(\sigma_{0} L_{0}\right)$. In order to understand the effect of loading rate on ductile crack initiation, dynamic analyses at two different values of $j(1.0$ and $2.0 \mathrm{GN} / \mathrm{ms})$ are plotted in figure $7 \mathrm{~b}$. It is evident from figures $7 \mathrm{a}, \mathrm{b}$ that the development of porosity in the ligament occurs slowly under dynamic loading, compared to the static case. For example, at a load level of $J /\left(\sigma_{0} L_{0}\right)=0.4$ (figure 7a), the void volume fraction is more than $5 \%$ over a considerable portion of the ligament in the static analysis. In contrast, figure $7 \mathrm{~b}$ shows that, at the same load level, the void volume fraction, at all points in the ligament (except, very near the notch tip and the hole), is much lower in the dynamic analyses. Furthermore, the porosity level in the ligament at a particular value of $J /\left(\sigma_{0} L_{0}\right)$ (figure $7 \mathrm{~b}$ ) decreases with the increase in loading rate, $\dot{j}$. Consequently, as discussed in the following section, the
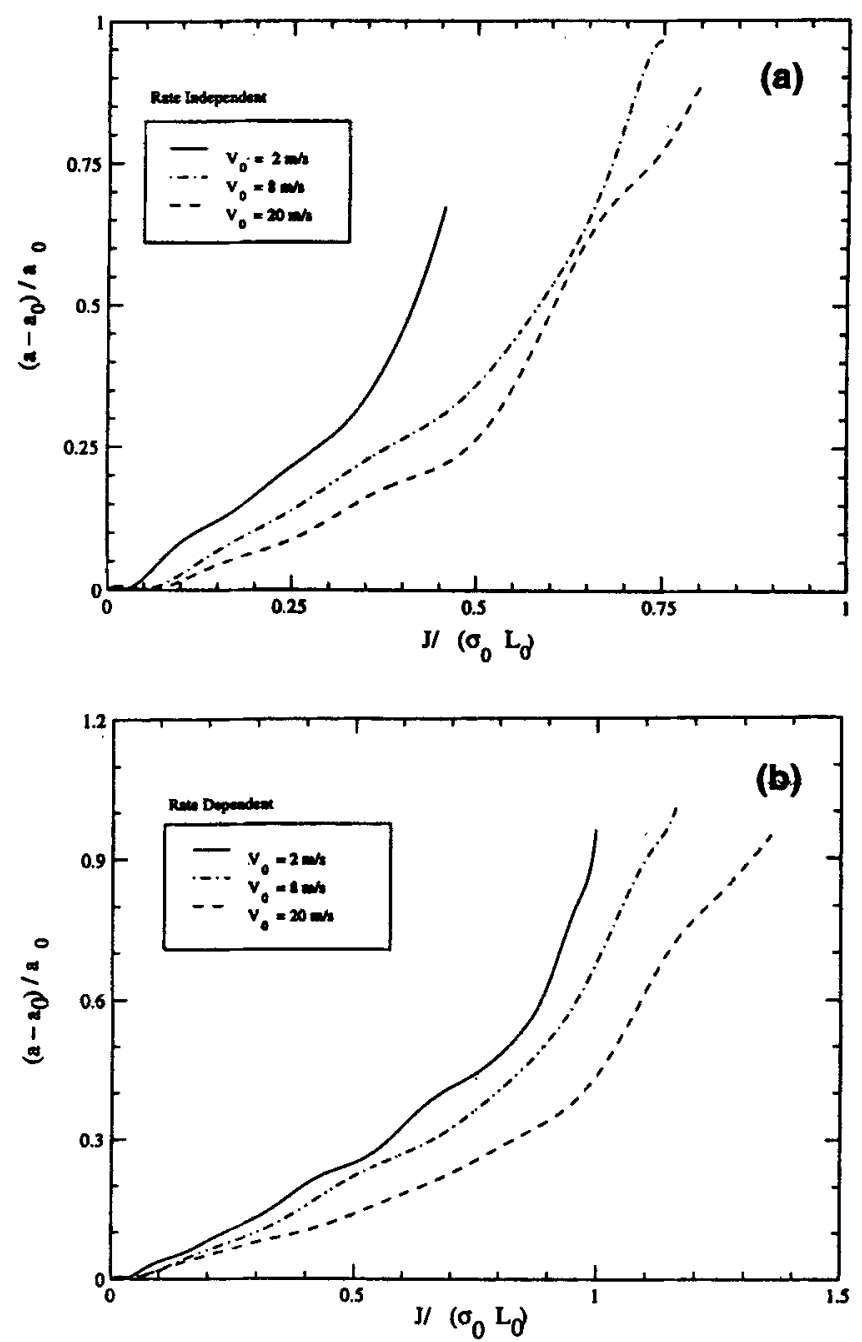

Figure 6. Variation of normalized hole opening with $J /\left(\sigma_{0} L_{0}\right)$ corresponding to different impact speeds applied on the TPB specimen for $\mathbf{a}$. rate-independent case, and $\mathbf{b}$. rate-dependent case. 
value of $J /\left(\sigma_{0} L_{\theta}\right)$, at which initiation of fracture occurs, is lowest for the static analysis, increasing progressively with the increase in the loading rate.

\subsection{Comparison of dynamic failure in two specimen geometries}

In figure 8 , the evolution history of the normalized transverse hole opening in the SEN(T) and TPB specimens have been compared at the same average loading rate of $j=0.8 \mathrm{GN} / \mathrm{ms}$. The results presented in this figure pertain to the rate-dependent case. It can be seen from this figure that, the evolution of hole growth near the notch tip in the two specimens, with respect to $J /\left(\sigma_{0} L_{0}\right)$, occurs in an almost identical manner when the notch tip region is loaded at the same average $j$. This
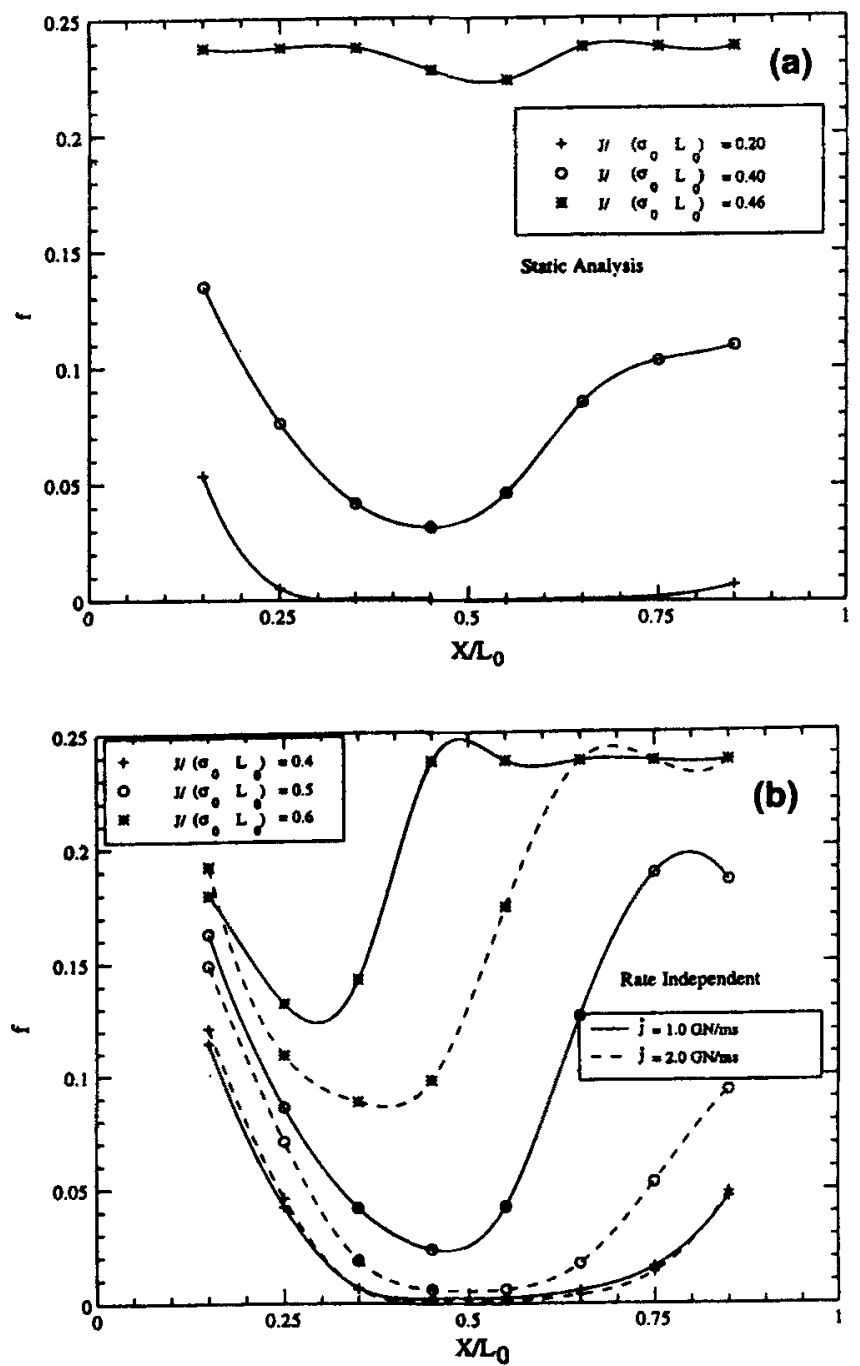

Figure 7. a. Distribution of $f$ in the ligament at various levels of $J /\left(\sigma_{0} L_{n}\right)$ obtained from rate-independent analysis of SEN(T) specimen pertaining to a. static loading, and b. dynamic loading at two different values of $\boldsymbol{j}$. close agreement is also reflected in the variation of the notch opening with respect to $J /\left(\sigma_{0} L_{0}\right)$, and porosity development in the ligament connecting the notch tip and the hole. In this context it should be emphasized that ratedependent material behaviour that allows for local flow stress elevation due to high strain rates, has been taken into account in this comparative study. These results suggest that the value of $J$ at fracture initiation (associated with complete failure of the ligament by micro-void coalescence), referred to as $J_{c}$, may be plotted for both the specimens as a function of $j$. In particular, it is important to examine whether the $J_{\mathrm{c}}$ vs $j$ locus is material dependent, rather than specimen-dependent to a large extent.

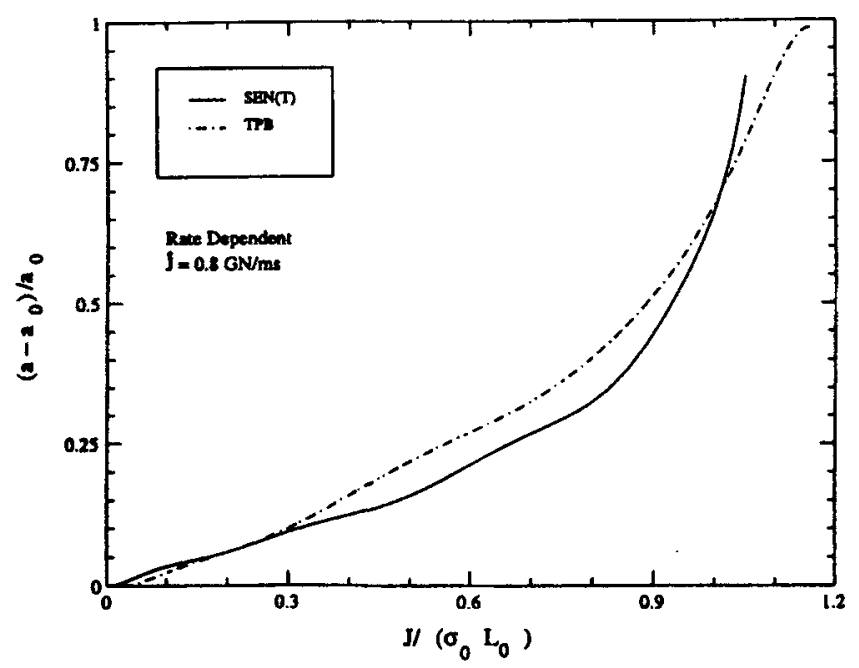

Figure 8. Comparison in the evolution histories of normalized hole opening with $J /\left(\sigma_{0} L_{0}\right)$ at a loading rate of $j=0.8 \mathrm{GN} / \mathrm{ms}$ for the SEN(T) and TPB specimen.

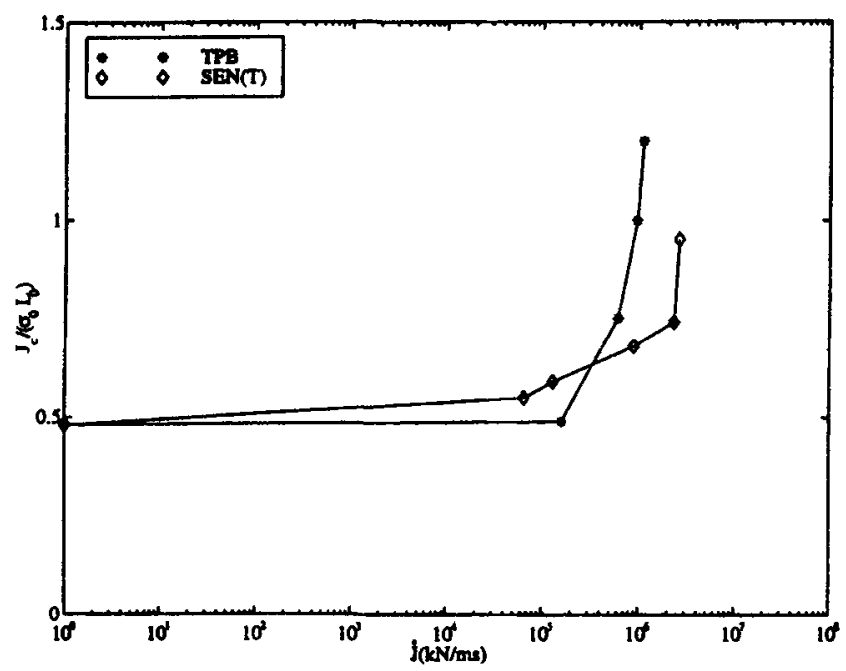

Figure 9. The locus of $J_{\mathrm{c}} /\left(\sigma_{0} L_{0}\right)$ with $j$ obtained from analysis of SEN(T) and TPB specimens corresponding to the rateindependent case. 


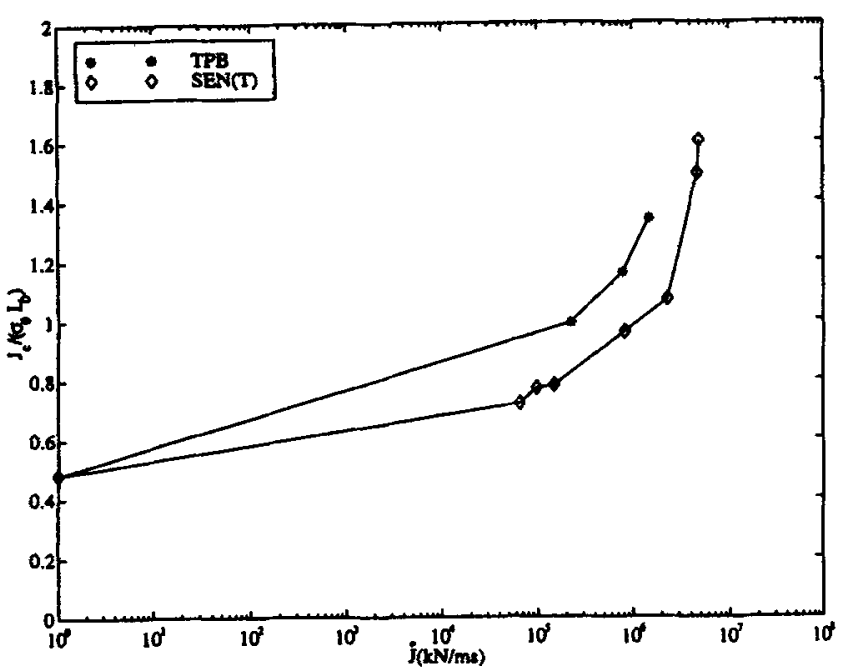

Figure 10. The locus of $J d\left(\sigma_{0} L_{0}\right)$ with $j_{\text {obtained from analysis }}$ of SEN(T) and TPB specimens corresponding to the ratedependent case.

In figure 9 , the variations of $J_{\mathrm{c}} /\left(\sigma_{0} L_{0}\right)$ with $j$ for the rate-independent material obtained from the analysis of the SEN(T) and TPB specimens have been plotted. Similar results for the rate-dependent case are presented in figure 10. The static value is also indicated in these figures in the limit of low loading rate $(j \sim 1 \mathrm{kN} / \mathrm{ms})$. It can be seen that somewhat higher loading rates are achieved by using the SEN(T) specimen compared to the TPB specimen (figures 9 and 10). Furthermore, for the rate-independent material, $J_{c} /\left(\sigma_{0} L_{0}\right)$ does not significantly differ from the static limit till a value of $\dot{j}$ of about $10^{5} \mathrm{kN} / \mathrm{ms}$ is attained (figure 9). However, for $j$ greater than $10^{5} \mathrm{kN} / \mathrm{ms}, J_{\mathrm{c}} /\left(\sigma_{0} L_{0}\right)$ increases rapidly with $j$, for both the specimens. This agrees with the recent experimental results of Owen et al (1997) for 2024 aluminium alloy. Moreover, the variation of $J_{\mathrm{c}} /\left(\sigma_{0} L_{0}\right)$ with $j$, obtained from the two specimens, is almost the same.

Furthermore, $J_{\mathcal{c}} /\left(\sigma_{0} L_{0}\right)$ for the rate-dependent material also shows a strong elevation for loading rates $\dot{j}$ greater than $10^{5} \mathrm{kN} / \mathrm{ms}$ (figure 10 ). However, for a given $j$, the value of $J_{c} /\left(\sigma_{0} L_{0}\right)$ for the rate-dependent case (figure 10) is higher than that for the rate-independent material (figure 9). Figure 10 also indicates that the variation of $J_{\mathfrak{c}} /\left(\sigma_{0} L_{0}\right)$ vs $j$, obtained from the analyses of the two specimens, are reasonably close with slightly higher values obtained for the TPB specimen.

\section{Conclusions}

The important conclusions from the numerical simulations reported in this paper can be summarized as:

(i) The ductile fracture processes of void growth and porosity development near a notch tip under dynamic loading are slower for a rate-dependent material compared to the rate-independent case.

(ii) Ductile fracture initiation is retarded as the loading rate increases. This is observed in both the SEN(T) and TPB specimens analyzed in this work.

(iii) A quantitative comparison of the results obtained from the SEN(T) and the TPB specimens at the same average loading rate $j$ shows that the evolution history of the notch opening and hole opening with respect to $J /\left(\sigma_{0} L_{0}\right)$ are closely identical. This is reflected in the porosity development in the ligament connecting the notch tip and the hole as well.

(iv) The critical value of $J$ at ductile fracture initiation shows very little elevation over the static limit for loading rates $j$ less than $10^{5} \mathrm{kN} / \mathrm{ms}$ and thereafter increases rapidly with loading rate. This is qualitatively similar to experimental results (see, for example, Owen et al 1997).

(v) For a given $\dot{j}$, the rate-dependent material displays a higher $J_{c} /\left(\sigma_{0} L_{0}\right)$.

(vi) The variation of $J_{c} /\left(\sigma_{0} L_{0}\right)$ with average loading rate $j$ predicted from the micro-mechanical modelling of the two specimens are quite similar, both for the rateindependent and rate-dependent material response. This suggests that experimental data for dynamic fracture initiation in ductile solids can be organized in the form of a $J_{\mathrm{c}}$ vs $j$ locus. This can be employed in fracture-based design of structural components that are subjected to impact or shock loading.

\section{References}

Andersson H 1977 J. Mech. Phys. Solids 25217

Aravas N and McMeeking R M 1985 Int. J. Fracture 2921

Argon A S, Im J and Safoglu R 1975 Metall. Trans. A6 825

Basu S and Narasimhan R 1996 Int. J. Solids Structures 33 1191

Basu S and Narasimhan R 1999a J. Mech. Phys. Solids 47325

Basu S and Narasimhan R 1999b Int. J. Fracture (submitted)

Belytshko T 1981 in Computational methods for transient analysis (eds) $\mathrm{T}$ Belytshko and $\mathrm{T} J \mathrm{R}$ Huges (Amsterdam: North Holland) p. 1

Brown L M and Embury J D 1973 in Microstructure and design of alloys. Proceedings of the third international conference on strength of metals and alloys (England: Cambridge Univ. Press) p. 164

Budiansky B, Hutchinson J W and Slutsky S 1982 in Mechanics of solids (eds) H G Hopkins and M J Sewell (England: Pergamon) p. 13

Chu C C and Needleman A 1980 J. Eng. Mater. Tech. 102249

Cox T B and Low J R 1974 Metall. Trans. 51457

Hutchinson J W 1968 J. Mech. Phys. Solids 1613

Nakamura T, Shih C F and Freund L B 1986 Eng. Fracture Mech. 25323

Narasimhan R 1994 Eng. Fracture Mech. 47919

Needleman A and Tvergaard V 1987 J. Mech. Phys. Solids 15 151

Needleman A and Tvergaard V 1991 Int. J. Fracture 4941 
Owen D M, Zhuang S, Rosakis A J and Ravichandran G 1997 Experimental determination of dynamic crack initiation and propagation fracture toughness in thin aluminum sheets, GALCIT Report, California Institute of Technology, Pasadena, USA

Pan J, Saje M and Needleman A 1983 Int. J. Fracture 21261

Pierce D, Shih C F and Needleman A 1984 Comput. Struct. 18 875

Priest A H 1976 in Proceedings of the international conference in dynamic fracture toughness (England: Cambridge) p. 95
Rice J R and Johnson M A 1970 in Inelastic behaviour of solids (eds) M F Kanninen, W F Adler, A R Rosenfield and R I Jaffee (New York: McGraw Hill) p. 641

Thomason P F 1990 Ductile fracture of metals (Oxford: Pergamon)

Tvergaard V 1981 Int. J. Fracture 17389

Tvergaard V 1982 Int. J. Fracture 18237

Tvergaard V and Needleman A 1984 Acta Metall. 32157

Tvergaard V and Needleman A 1988 Int. J. Fracture 37 197 